\title{
Le commerce mondial et les exigences de normalisation : réorganisation locale et repositionnement global des systèmes agro-alimentaires. Le cas des agrumes de la Comunidad Valenciana (Espagne)
}

\section{The international trade and standardisation requirements : local reorganisation and global repositioning of agrofood systems. The case of citrus system from the Valencian Community (Spain)}

\author{
Juan R. Gallego-Bono* \\ Departament d'Economia Aplicada, Universitat de València, Avinguda dels \\ Tarongers s/n 46022 Valencia, España.
}

\section{Résumé}

L'évolution du commerce international et les exigences de standardisation ont conduit les systèmes agro-alimentaires à se réorganiser. Cet article éclaire trois aspects du processus de réorganisation de l'agrumiculture de la Comunidad Valenciana: la modification des relations inter-entreprises ; les évolutions au sein du système d'innovation ; l'intensification des interactions des opérateurs valenciens avec le secteur agrumicole d'Argentine et d'Uruguay. S'appuyant sur une approche évolutionniste et une méthodologie qualitative basée sur la réalisation d'entretiens personnels approfondis, l'article montre que cette réorganisation est le produit d'un processus social et ouvert qui dépend de l'histoire, des

*Adresse email : Juan.R.Gallego@uv.es

doi:10.3166/ges.9.329-343 (C) 2007 Lavoisier, Paris. Tous droits réservés. 
compétences et des stratégies de l'ensemble des acteurs. En ce sens, il met en exergue le fait que, si les normes favorisent un rapprochement entre les différents acteurs au sein d'un même système ou entre systèmes différents, elles peuvent également s'avérer des obstacles en termes de communication.

(C) 2007 Lavoisier, Paris. Tous droits réservés.

\section{Summary}

Trends in international trade and standardisation requirements have made it necessary to carry out thorough re-organisation of agrifood systems. This article analyses the process of re-organising the production and innovation systems in the citrus fruit sector in the Valencian Community, as well as interaction with the citrus fruit sector in Argentina. An evolutionary approach and qualitative methodology are adopted, on the basis of in-depth personal interviews. The article shows that the re-organisation is an open, social process which depends on the background, competencies and strategies of those involved. Standards are seen to promote cohesion, both within and amongst agrifood systems, although they may also represent obstacles to communication.

(C) 2007 Lavoisier, Paris. Tous droits réservés.

Mots clés : Systèmes agro-alimentaires, systèmes régionaux d'innovation, normalisation, institutions, réseaux.

Keywords: Agro-food systems, regional innovation systems, normalisation, institutions, networks.

Fort d'une longue tradition exportatrice, le secteur valencien des agrumes a été pleinement touché par ce phénomène. L'Espagne est le cinquième producteur mondial d'agrumes, avec $6 \%$ de la production mondiale et une spécialisation dans le domaine des fruits frais. La Comunidad Valenciana se distingue par sa spécialisation dans la mandarine et par son haut niveau d'exportation (Gallego, 2004). Elle est en effet le premier exportateur mondial de fruits frais avec une part de marché d'environ $30 \%$, les mandarines (autour de 50\% des exportations mondiales) et les oranges (28\% des exportations mondiales) représentent un poids important (FAO, 2003 et 2005). Par conséquent, il est clair que les changements récents d'exigences des consommateurs (qualité, sécurité alimentaire et respect de l'environnement), et l'importance prise par les chaînes de supermarchés dans la distribution des produits frais, ont particulièrement touché le secteur agrumicole valencien. Nous verrons que ces évolutions ont nécessité un processus de standardisation des pratiques agricoles et du traitement des produits frais, tant au niveau de la production qu'en ce qui concerne les stations de conditionnement et le transport.

Les systèmes agro-alimentaires constituent un cadre propice pour étudier les institutions et les relations, tant formelles qu'informelles (Torre, 2000), qui favorisent la création de ressources spécifiques à un territoire et le développement de capacités d'adaptation. La standardisation représente un enjeu global (Held, 2005) qui implique que l'adaptation des systèmes agro-alimentaires dépasse le seul cadre local pour s'articuler 
avec d'autres systèmes régionaux, nationaux et internationaux. L'article vise à analyser dans quelle mesure le processus de réorganisation interne propre au système agrumicole valencien s'articule avec l'importation d'oranges en contre-saison d'Argentine.

Les analyses développées dans l'article s'appuient sur un schéma conceptuel évolutionniste combinant les approches des systèmes régionaux d'innovation (Cooke et al., 1998) et des réseaux technico-économiques (Callon, 1991). Dans cette perspective, l'innovation dépend de l'interaction de différents acteurs avec certaines institutions, activités ou éléments matériels existant sur le territoire (ou en interaction avec d'autres territoires) qui contribuent à mettre en rapport ces acteurs. L'innovation est alors un processus de création sociale, dans le sens où il s'agit d'un processus d'interaction et de constitution de réseaux entre des acteurs hétérogènes, mais dans le sens également où, en retour, la nature de ces réseaux va conditionner le processus innovateur. En second lieu, nous supposons que les normes et les standards augmentent la connectivité (Metcalfe, 1995) et les capacités systémiques (Kaufmann et Tödtling, 2001) d'acteurs hétérogènes aussi bien à l'intérieur des différents systèmes qu'entre ceux-ci, en fournissant des points de référence partagés pour communiquer, la communication étant considérée comme la base de la créativité. L'importance de ces références communes s'appuie sur l'hypothèse selon laquelle la proximité géographique (en termes de distance-temps) ne suffit pas et n'est même pas indispensable pour innover, même si elle favorise a priori l'interaction. Il est nécessaire qu'existe également une proximité organisationnelle, c'est-à-dire relationnelle, qui repose sur toute une série de règles et de normes sociales et techniques de comportement partagées par les acteurs (Rallet et Torre, 2005).

L'article est organisé de la façon suivante. La première partie caractérise le processus interne de réorganisation du système de production des agrumes de la Comunidad Valenciana et de son système d'innovation. En focalisant sur le réseau des relations entre les entreprises et les institutions sur des aspects particuliers, nous verrons que les exigences de standardisation associées au processus de mondialisation d'un secteur très marqué par l'exportation ont stimulé la professionnalisation et le développement de nouvelles compétences dans les centrales agrumicoles. La deuxième partie analyse le processus d'internationalisation de ce système et, plus particulièrement, ses interactions avec l'Amérique Latine, à l'occasion de l'importation d'oranges en contre-saison. Ce phénomène est orienté par les acteurs locaux les plus dynamiques. Nous verrons que la montée des standards a eu pour conséquence de générer un langage partagé stimulant la communication, les synergies et la mise en place de relations stables entre des acteurs des différents systèmes territoriaux. Cependant, les caractéristiques politiques et stratégiques des acteurs ont été à l'origine de barrières en termes de communication. De fait, on observe une certaine segmentation des différents systèmes territoriaux qui est un facteur limitant la portée de la mondialisation et des échanges.

\section{Méthodologie}

L'analyse repose sur la réalisation de 101 entretiens personnels approfondis. Ils ont été réalisés auprès de deux types différents d'acteurs des provinces de Castellón et de Valencia pendant la période 2002-2006. Ces entretiens concernent, d'une part, 87 entreprises de la chaîne de valeur (c'est-à-dire intégrant l'ensemble des sous-secteurs reliés entre eux par leur contribution à la création d'un même produit), soit 27 centrales agrumicoles, 
31 fournisseurs et 29 agriculteurs. Les entretiens ont été réalisés à l'aide d'un questionnaire fermé. L'échantillon d'entreprises a privilégié les unités les plus novatrices mais tient compte de la diversité de la population totale composée d'environ 400 centrales agrumicoles, 20 fournisseurs de machines et de produits chimiques post-récolte, 20 pépiniéristes, 15 fabricants et 100 distributeurs de produits agro-chimiques et de fertilisants. D'autre part, 14 entretiens ont été réalisés avec les directeurs de toutes les organisations composant le «Réseau des Agrumes » de la Comunidad Valenciana. Ce dernier regroupe les principaux membres du système institutionnel d'innovation de la culture des agrumes de la Comunidad Valenciana (ci-après désigné par SICI). Il se caractérise par une grande variété d'acteurs : centres de recherche (Institut Valencien de Recherches Agricoles (IVIA), Institut d'Agrochimie et de Technologie des Aliments, etc.), divers centres techniques (Service de Développement Technologique Agricole, etc.), une Interprofession (Intercitrus), une association d'entreprises (Institut Technologique Agro-Alimentaire) ainsi que des associations privées (Groupements des Pépiniéristes d'Agrumes, Comité de Gestion pour l'Exportation d'Agrumes). Dans ce cas, les questionnaires étaient semiouverts et conçus de façon à permettre la comparaison des résultats avec ceux recueillis dans les entreprises.

\section{Exigences de standardisation et réorganisation interne du système agrumicole valencien}

\section{1 Les acteurs du secteur agrumicole valencien}

En 2003, 80.436 exploitations produisaient des agrumes dans la Comunidad Valenciana, représentant une superficie totale de 172.625 hectares. La production annuelle d'agrumes était de 3.833 tonnes, soit $61,5 \%$ de la production espagnole, répartie entre les oranges $(46,2 \%)$, les mandarines $(44,7 \%)$ et les citrons $(9,1 \%)$. La même année, $69,5 \%$ de la production d'agrumes étaient exportés pour $53 \%$ en moyenne nationale. Le nombre de personnes travaillant dans le secteur agrumicole en pleine saison est estimé à environ 100000.

Ce système, très fortement orienté vert l'exportation, s'est organisé progressivement au cours du $\mathrm{XX}^{\mathrm{e}}$ siècle sur le principe d'une division sociale du travail, le long d'une chaîne de valeur qui va de la production agricole jusqu'à la commercialisation, et a abouti à la constitution d'un réseau de relations entrepreneuriales. La chaîne de valeur des agrumes de la Comunidad Valenciana se compose d'un grand nombre de sous-secteurs spécialisés qui ont développé des relations marchandes et non marchandes, localisées sur le territoire (Gallego 2004).

$\mathrm{Au}$ sein de cette division, les centrales agrumicoles constituent un acteur stratégique puisqu'elles agissent en tant que passerelle entre la distribution commerciale et les agriculteurs. Il s'agit d'entreprises qui achètent le produit aux agriculteurs-propriétaires, se préoccupent de le conditionner et de l'emballer dans les stations de conditionnement puis le commercialisent. Ces centrales agrumicoles peuvent être des entreprises privées ou des coopératives. Les coopératives commercialisent la production de leurs associés (des producteurs), elles leur fournissent des inputs (fertilisants, etc.) et des services à la production. Ce rôle est d'autant plus important que la production est essentiellement réalisée par de très petites exploitations (minifundisme). 
Les fournisseurs constituent un autre maillon important de la chaîne de valeur. Une partie livre des produits aux producteurs. Produites par les pépiniéristes, les plantes sont les inputs essentiels des agriculteurs, au même titre que les produits chimiques (fertilisants, pesticides, etc.) que ceux-ci achètent aux entreprises de distribution et aux coopératives qui commercialisent les produits fabriqués par des multinationales. Une autre partie fournit les centrales agrumicoles. Il s'agit des fabricants de machines des stations de conditionnement (machines à calibrer, pour la mise en caisse, pour le conditionnement, etc.) ou des produits chimiques post-récolte (cires, produits fongicides, etc.), qui conçoivent et fabriquent également les machines permettant l'application de ces produits et, enfin, des fabricants d'emballages. Finalement, en matière de transport, il existe une tendance à ce que les entreprises spécialisées s'occupent de transporter les produits depuis les centrales agrumicoles jusqu'aux marchés ou aux centres de distribution, bien qu'il existe certaines centrales qui possèdent leurs propres moyens de transport ou qui ont des participations dans des entreprises de transport.

Hormis les fabricants de fertilisants et de pesticides, les entreprises de la chaîne de valeur sont essentiellement valenciennes, que ce soit par leur origine ou parce qu'elles s'y sont complètement insérées. Les principaux fabricants de pesticides ont des délégations dans la région et essayent de resserrer leurs liens avec le territoire.

\subsection{L'adaptation du système de production valencien dans le processus de standardisation : intensification des relations et innovation}

La montée de nouvelles exigences de la part des consommateurs et des grandes chaînes de distribution en matière de qualité, de respect de l'environnement et de sécurité alimentaire ont entraîné, depuis une quinzaine d'années, une transformation progressive du système agrumicole. Comme nous allons le voir, ce processus a trois principales implications: 1) un changement dans les relations entre les différentes entreprises de la chaîne de valeur; 2) un changement des relations entre les entreprises et les centres de recherche et 3) une intensification de l'internationalisation du système valencien des agrumes.

La standardisation consiste dans la mise en place d'un ensemble de règles et de paramètres, publics et privés, dont le but est d'établir des références objectives et prédéterminées pour l'action. Elle intègre des éléments tels que la normalisation des produits (calibres, couleur, goût), des règles en matière de pratiques culturales, de conditionnement et de transport. Elle suppose l'assignation de responsabilités aux différents acteurs qui participent à une tâche et couvre différents domaines, depuis des questions techniques jusqu'à des questions de qualité, d'environnement, de sécurité alimentaire et d'éthique du travail (Mercier et Tanguy, 2005). Ces exigences croissantes en matière de respect des standards et normes publics («Analyse des risques et des points critiques de contrôle ») et privés («EUREPGAP», «Nature Choice », BRC, etc.) ont contraint les centrales agrumicoles à s'inscrire dans le cadre des «bonnes pratiques agricoles », à en vérifier la mise en œuvre et à assurer la traçabilité des produits à l'intérieur des stations de conditionnement. Ce processus a nécessité l'embauche de techniciens (ingénieurs agronomes, biologistes, économistes, etc.) dans les centrales à partir du début des années 1990. De même, il a contribué à une intensification des relations entre les centrales agrumicoles et les agriculteurs, par la voie du conseil dispensé auprès des producteurs par les centrales. Dans le même temps, les 
entreprises locales de distribution de fertilisants et de produits agro-chimiques aux agriculteurs ont elles-mêmes commencé à leur proposer des conseils techniques. Ainsi, dans ce système, les centrales agrumicoles et les entreprises offrant des inputs et des services agricoles, sont les pourvoyeurs des renseignements techniques auprès des agriculteurs.

Les centrales agrumicoles travaillent avec les agriculteurs sur la base de « cahiers des charges » indiquant la liste des produits chimiques autorisés pour les traitements phytosanitaires et les doses correctes à appliquer. Les agriculteurs doivent noter toutes les opérations qu'ils réalisent et peuvent faire appel au conseil des techniciens de la centrale. Ainsi, la relation entre cette dernière et les agriculteurs présente un caractère stable et acquiert une dimension technique. Elle a constitué une voie de diffusion systématique de connaissances, inédite jusqu'à présent. Ce rapport technique est particulièrement intense dans le cas des coopératives dans la mesure où elles assument, pour le compte de leurs associés (adhérents), un certain nombre de fonctions qui auparavant étaient prises en charge par les agriculteurs eux-mêmes ou par des spécialistes. On assiste ainsi à un mouvement à la fois de technification des cultures, d'intensification de la division sociale du travail et d'organisation entrepreneuriale des tâches agricoles.

Mais le progrès technique des centrales ne pouvait pas s'accomplir seulement par l'amélioration interne. Une intensification des relations avec les fabricants d'équipements et de produits chimiques post-récolte a aussi été nécessaire. Il existait déjà, dans la Comunidad Valenciana, un lien relativement étroit entre les centrales et leurs fournisseurs. Cependant, les exigences croissantes de précision et de diversité en matière de calibres, la multiplication des conditionnements des produits et le besoin d'assurer leur traçabilité ont impliqué de nouvelles demandes et la nécessité d'une coopération croissante entre les sociétés de ces différents sous-secteurs.

Notre enquête a montré qu'il en est résulté un apprentissage interactif. Ce processus se traduit par une participation intense des usagers dans le design et l'adaptation des machines. De même, les limitations concernant les matières actives autorisées pour l'élaboration de produits post-récolte (cires, fongicides et détergents), le besoin de contrôler et de minimiser les déchets et les fruits pourris, le besoin de concevoir des nouvelles techniques de conservation, qui préservent certaines propriétés des produits et respectent l'environnement et la sécurité du point de vue alimentaire, ont entraîné de nouvelles demandes des entreprises agrumicoles à leurs fournisseurs de post-récolte. Ces collaborations ont permis la fabrication de machines et de produits chimiques adaptés à la réalisation des objectifs en termes de qualité, de minimisation de déchets, etc.

Plus largement, cet ensemble d'interactions est devenu le garant du respect de la réglementation publique et privée par les centrales agrumicoles et, par conséquent, par les agriculteurs eux-mêmes. Grâce à l'information stratégique (sur les marchés) dont disposent les centrales agrumicoles et grâce à un meilleur équipement matériel et une collaboration avec le personnel (R\&D, technique) des fournisseurs, c'est aussi l'une des principales sources d'innovation du système dans son ensemble. Ainsi, la centrale agrumicole peut-elle s'engager à acquérir les produits de son fournisseur (cire, en particulier) à un prix fixé et, en échange, elle se voit offrir par son fournisseur un service de conseil technologique et organisationnel, une assistance technique (entretien des machines), la réalisation d'essais, des contrôles qualité et voire, comme l'ont montré nos entretiens les plus récents, la mise à disposition de personnel susceptible d'occuper 
des postes techniques et/ou de gérant, d'une haute responsabilité. Cette relation est favorisée par la présence d'agents commerciaux de post-récolte disposant d'une solide formation technique (en chimie, biologie ou génie).

En définitive, nous observons que le développement des normes et des standards a stimulé la réorganisation des relations intersectorielles tout au long de la chaîne de valeur. Cette réorganisation a été rendu possible par la proximité organisationnelle (technique), institutionnelle et culturelle que les entreprises des différents sous-secteurs entretiennent (Rallet et Torre, 2005). Elle a elle-même été favorisée par les relations entre les techniciens. Les normes et les standards ont créé un ensemble de référentiels partagés par des personnes appartenant à des organisations différentes. La division sociale du travail et la coopération intersectorielle se sont intensifiées. L'apprentissage a été mutuel dans la mesure où les fournisseurs d'équipements et de produits chimiques ont pu acquérir, par le biais de ces collaborations, des connaissances importantes leur permettant de développer une stratégie d'internationalisation et de mondialisation.

\subsection{Le système institutionnel d'innovation ou l'importance des organismes publics pour le développement du secteur}

Du point de vue institutionnel, le système d'innovation de la culture valencienne des agrumes (SICI) repose sur trois axes ${ }^{1}$.

Le premier axe est constitué d'un ensemble d'organismes rattachés au Conseil de l'Agriculture, de la Pêche et de l'Alimentation du Gouvernement Valencien (CAPA) : l'Institut Valencien de Recherche Agricole (IVIA), qui est un des principaux centres de recherche appliquée du monde en matière de culture d'agrumes; le Service du Développement et la Technologie Agricole (SDT) et le Département de Protection des Cultures (APC). Le SDT, étroitement lié à l'IVIA, est spécialisé dans le développement expérimental et s'affirme comme le principal centre de diffusion d'innovations et de formation des agriculteurs en matière de culture. L'APC remplit cette même fonction en ce qui concerne les fléaux et les maladies, en dirigeant leur contrôle effectif sur le terrain, et s'occupe également du contrôle de la qualité et de la certification du matériel végétal.

Le deuxième pilier du système institutionnel d'innovation se fonde sur le Département de la culture des Agrumes et le Département d'Entomologie de l'Université Polytechnique de Valencia (UPV). C'est le principal centre de formation d'ingénieurs agronomes de la Comunidad Valenciana. Le premier, spécialisé dans la culture, s'appuie sur le Service du Développement et la Technologie Agricole du gouvernement pour arriver jusqu'aux techniciens et jusqu'aux agriculteurs. De la même façon et à ces mêmes fins, le Département d'Entomologie s'appuie sur le Département de Protection des Cultures.

\footnotetext{
1 Nous pourrons mentionner les institutions et centres de formation, d'innovation et/ou de transfert technologique grâce à leur abréviation: 1) le Conseil de l'Agriculture, de la Pêche et de l'Alimentation (CAPA) du Gouvernement Valencien; 2) la Direction Générale de l'Innovation et de l'Elevage (DGI); 3) l'Institut Valencien des Recherches Agricoles (IVIA); 4) le Service du Développement et la Technologie Agricole (SDT); 5) le Département de Protection des Cultures (APC); 5) le Département de la Culture des Agrumes et le Département d'Entomologie de l'Université Polytechnique de Valencia (UPV); 6) l'Institut de Biologie Moléculaire et Cellulaire des Plantes (IBMCP); 7) l'Institut Technologique Agro-alimentaire (AINIA); 8) 1'Institut d'Agrochimie et de Technologie des Aliments (IATA); 9) l'Institut pour la Petite et la Moyenne Entreprise Valencienne (IMPIVA).
} 
Un troisième pilier du SICI, enfin, est composé de l'Institut Technologique Agro-alimentaire (AINIA) et de l'Institut d'Agrochimie et de Technologie des Aliments (IATA). Ces deux centres ont pour vocation la technologie de post-récolte, bien qu'aucun des deux ne soit spécialisé dans le domaine des fruits frais mais plutôt dans celui de la transformation industrielle et de la technologie des aliments. L'AINIA fait partie du Réseau des Instituts Technologiques de l'IMPIVA (Gouvernement Valencien), mais il s'agit d'une association privée dont l'objectif est la prestation de services technologiques aux entreprises. L'IATA (et plus particulièrement l'équipe qui, au sein de l'IATA, a en charge la post-récolte) fait de la recherche fondamentale orientée et a pour objectif principal de réaliser des progrès scientifiques.

$\mathrm{Au}$ cours du processus de réorganisation du système agrumicole, le système institutionnel d'innovation s'est redéployé grâce à trois vecteurs essentiels.

Premièrement, l'embauche de techniciens par les centrales agrumicoles a permis à ces dernières de disposer d'interlocuteurs pertinents et favorisé, par leur intermédiaire, la diffusion d'innovations et d'informations directes et continues sur les différents problèmes agricoles. La présence de ces acteurs est une condition nécessaire à l'accès des entreprises aux compétences des centres de recherche $(\mathrm{CR})^{2}$.

Deuxièmement, dans le cadre d'une politique de modernisation et de rationalisation de la production, le Conseil de l'Agriculture, de la Pêche et de l'Alimentation (CAPA) a encouragé (surtout dans les coopératives) l'intégration de techniciens. En matière de contrôle technique des fléaux, ces techniciens dépendent du CAPA. Ils sont formés et/ou recyclés par des professeurs et des spécialistes de l'UPV et du CAPA. C'est un mécanisme essentiel dans l'instauration de liens de confiance et le développement de connaissances mutuelles. La recherche de l'IVIA et des départements de l'UPV, le développement expérimental du SDT et de l'APC, se réalisent dans des champs d'expérimentation appartenant à des coopératives, ce qui facilite la création de cette proximité entre les différents acteurs. Le CAPA a encouragé la création de réseaux d'innovation entre les institutions liées à la sphère de production et a intensifié l'orientation traditionnelle de l'IVIA dans cette branche. La recherche réalisée par cet organisme va du matériel végétal jusqu'à la mise sur le marché du produit frais, prêt à la consommation. L'amélioration génétique des variétés et des plants d'agrumes et l'élimination de pathogènes du matériel végétal avaient été jusqu'alors un domaine prioritaire de la recherche de l'IVIA ${ }^{3}$. Cette relative focalisation des ressources sur les premières phases du processus agricole est en partie responsable du développement plus limité de la recherche appliquée à la post-récolte des agrumes dans la Comunidad Valenciana.

Troisièmement, la prestation plus ou moins importante de services aux entreprises ${ }^{4}$ fournis par les centres de recherche est un aspect essentiel du système. En effet, plus que la demande de collaborations en matière de R\&D, l'établissement de nouvelles normes et standards a supposé une augmentation considérable de la demande de services aux CR,

\footnotetext{
2 De manière à simplifier l'exposé, tous les centres publics de formation, de recherche scientifique et technologique et de diffusion des innovations seront appelés centres de recherche (CR).

3 L'introduction de nouvelles variétés a constitué l'orientation privilégiée de l'innovation pour l'agriculteur et l'exportateur valencien. C'est une caractéristique du système agrumicole et un facteur de son dynamisme.

4 L'IATA, l'IBMCP, l'IVIA et le Département d'Entomologie de l'UPV, à la différence de l'AINIA et de l'autre Département de l'UPV, ne proposent pas de services au sens strict.
} 
notamment en termes de formation, d'analyse des produits finis et de déchets, de conseil et de préparation pour obtenir les certifications de qualité et d'environnement. Ces collaborations ont abouti à une intensification des relations personnelles entre les entreprises (techniciens) et les CR, par le biais des consultations par téléphone, des contacts personnels, etc.

Comme le rappelle la littérature sur les systèmes de production dans les secteurs traditionnels (Porter, 1991 ; Tomás Carpi et al ., 1999), les résultats de nos enquêtes montrent que ce sont les fournisseurs de produits destinés à la production (pépiniéristes et fabricants de fertilisants, pesticides, etc.) et de produits destinés à la phase de la post-récolte (fabricants d'équipements et de produits chimiques) qui ont le plus de relations avec les institutions de recherche et de transfert de technlogie (Gallego, 2004). Logiquement, les principaux clients en R\&D sont les sous-secteurs les plus intensifs en R\&D. Malgré cela, les compétences relatives plus limitées des CR dans le domaine de la post-récolte, une politique publique qui fait prévaloir la recherche dans le domaine de la production et des difficultés de collaboration avec les entreprises concernées font que, dans la région étudiée, la relation n'est pas si étroite qu'on pourrait le penser.

Dans ce système d'innovation, cependant, les demandes de services et les relations personnelles ont participé au rapprochement des centres de recherche avec de nombreuses entreprises qui n'ont pas d'activité de R\&D. Elles représentent un élément clé de la diffusion des innovations. Par le biais de ces demandes, les CR peuvent diffuser un ensemble d'innovations générées à travers les relations de R\&D avec les entreprises plus novatrices. Ce mécanisme vient compléter le rôle que jouent les fournisseurs de postrécolte. En effet, grâce à leur relation continuelle avec les usagers, ils peuvent diffuser une partie des innovations générées à travers une étroite interaction avec les centrales agrumicoles leaders (privées et coopératives) et les CR.

\section{L'internationalisation croissante des centrales agrumicoles de la Comunidad Valenciana}

\subsection{Le développement des importations à contre-saison}

Les exigences des marchés globaux et de maintien de leur compétitivité au niveau international ont progressivement conduit les entreprises (les centrales agrumicoles privés $^{5}$ ) à développer de nouveaux horizons et à rompre avec la saisonnalité de la production d'oranges fraîches.

Jusqu'en 1993, les importations d'agrumes étaient interdites en Espagne, à cause d'un ensemble de normes phytosanitaires. Cette situation condamnait les centrales agrumicoles valenciennes à la saisonnalité à cause de l'absence de production interne pendant les mois d'été. Les entreprises essayaient de pallier cette lacune en stockant dans des chambres froides des oranges de la variété «Valencia-Late » et en les écoulant de façon progressive sur le marché intérieur entre juillet et octobre. Cette solution entraînait des problèmes de qualité malgré les progrès technologiques en matière de conservation. D'autre part, étant

5 Jusqu'à récemment, les centrales agrumicoles coopératives valenciennes ne réalisent pas d'importations directes en contre-saison. En conséquence, l'évocation de ce type d'importations se réfère aux seules entreprises privées. 
donné que les pays habituels de destination des exportations espagnoles (la France en particulier) importaient des oranges de l'hémisphère sud (notamment de l'Amérique du Sud et de l'Afrique du Sud) en contre saison, les exportateurs valenciens perdaient toute prise sur ces marchés.

Avec la mise en place du Marché Unique Européen en janvier 1993, l'Espagne se trouve dans l'obligation d'ouvrir ses frontières aux agrumes provenant de pays étrangers. Le marché espagnol devient alors un objectif potentiel pour l'ensemble des opérateurs mondiaux (importateurs intermédiaires, centrales agrumicoles, etc.) intervenant dans l'orange de contre-saison. C'est cette crainte qui conduit, dans un premier temps, les centrales agrumicoles espagnoles et valenciennes à réaliser des importations à contre-saison. Puis, d'une manière plus progressive et spécifique, ce sont les exigences des clients, les pressions de la concurrence et le besoin de rationalisation du coût des installations de production, qui amènent les entreprises valenciennes privées et, notamment, celles de plus grandes dimensions, à s'engager dans cette activité. Les importations en contre-saison ont ainsi permis d'offrir un produit frais et sûr, de fidéliser les clients toute l'année, de réduire les coûts par unité de produit et d'accélérer l'amortissement des installations.

\subsection{Une ouverture vers l'Amérique Latine favorisée par des normes communes}

Pour réaliser leurs importations à contre-saison, les centrales agrumicoles valenciennes se sont tournées vers l'Argentine pour des raisons à la fois économiques, géographiques et culturelles. Depuis le début des années 1990, les relations entre centrales valenciennes et argentines ont connu deux phases bien distinctes : une première phase de croissance de la confiance et d'intensification des échanges; depuis la fin des années 90, une seconde phase marquée par la méfiance et la réduction des relations directes entre les producteurs des deux pays.

Un des principaux moyens d'accès aux entreprises de l'hémisphère sud provient des contacts entretenus par les fournisseurs valenciens de produits de post-récolte déjà implantés en Argentine. Une autre voie d'approche tient à la demande des clients des entreprises valenciennes (les chaînes de distribution) qui leur ont « suggéré » de prendre contact avec les producteurs argentins. Enfin, la ressemblance entre les variétés d'oranges argentines et les variétés valenciennes a facilité le rapprochement d'un nombre croissant de centrales agrumicoles valenciennes avec des centrales agrumicoles d'Argentine, d'Uruguay et d'Afrique du Sud.

Ce processus d'importation directe est très significatif parce qu'il a impliqué la réorientation vers l'Espagne (spécialement vers la Comunidad Valenciana) d'une partie importante des exportations d'oranges d'Amérique Latine, et de l'hémisphère sud en général, qui préalablement étaient dirigées vers d'autres pays européens.

La contrepartie directe de la stagnation relative et/ou de la baisse des importations hollandaises, allemandes, françaises et anglaises d'oranges de l'hémisphère sud, et surtout d'Amérique Latine, est l'augmentation des importations espagnoles provenant de l'hémisphère sud et des exportations espagnoles vers les pays européens. Cela n'empêche pas que certaines plateformes logistiques traditionnelles d'importation et de distribution depuis d'autres pays de l'Union Européenne ont continué d'opérer. En fait, même si les importations valenciennes en contre-saison sont nées et sont essentiellement dirigées vers le marché intérieur, les exportations espagnoles d'une partie de celles-ci gagnent continuellement du terrain et bénéficient encore de marges de progression en termes de parts de marché. 
L'essentiel est que l'importation directe par les entreprises valenciennes est avantageuse, tant pour les importateurs que pour les exportateurs. Elle est le ferment d'un rapport étroit de coopération prenant la forme d'une joint venture informelle. Les responsables valenciens se déplacent fréquemment afin de connaître et d'essayer de faire le choix sur place des champs d'origine des oranges d'Amérique Latine et échangent des points de vue sur les conditions de production et sur les traitements. Cette importation s'accompagne de la mise en place d'une programmation qui comprend un calendrier d'expéditions, la fréquence de celles-ci, le calibre et le type d'expéditions. Les relations se produisent dans un contexte de transparence entre les parties, où l'exportateur et l'importateur échangent des informations sur les coûts et les marges bénéficiaires et où le prix réglé est fonction de la meilleure cotation atteinte sur le marché par la variété d'orange correspondante. Cette relation est garante de la fiabilité de son produit pour l'importateur espagnol et, pour l'exportateur argentin, d'une réduction de l'incertitude, de meilleurs prix et quantités vendues, par rapport aux conditions associées à la vente marchande à travers des intermédiaires et/ou aux enchères dans les ports d'arrivée et de distribution de l'orange. Pour comprendre cette transparence des relations, il est nécessaire de prendre en considération que la centrale agrumicole valencienne, à travers les importations d'oranges à contre-saison, cherche surtout à offrir un service au client et non pas à obtenir un éventuel bénéfice maximum, comme c'est le cas lorsque certaines de ses variétés sont commercialisées en pleine campagne.

Les centrales agrumicoles peuvent aussi opérer en tant que sous-traitantes ou entreprises de services d'une grande chaîne de supermarchés. Dans ce cas, les entreprises valenciennes se limitent à préparer les oranges réellement importées par une centrale d'achat d'une grande chaîne de supermarchés anglaise ou française en échange d'une redevance établie par palette. Cette modalité comprend parfois la création d'une entreprise mixte entre l'entreprise espagnole et l'entreprise argentine. Ce type d'accord a lieu entre les grandes centrales agrumicoles valenciennes et argentines. Dans ce cas, la coopération et l'échange d'informations sont beaucoup moins intenses parce que c'est la chaîne de supermarchés qui établit les conditions à respecter, tant pour les entreprises valenciennes que pour les argentines.

En ce qui concerne les mécanismes d'échange de connaissances, il faut souligner le rôle central joué par les normes et les standards dans le cadre des relations entre les entreprises. Premièrement, les centrales agrumicoles valenciennes n'ont aucun problème par rapport aux normes et aux standards avec leurs fournisseurs d'oranges de l'hémisphère sud parce que ceux-ci s'appuient sur une grande tradition exportatrice vers l'Europe (Mercier et Tanguy, 2005) et sur un niveau de certification supérieur ou égal à celui des entreprises valenciennes, processus favorisé par la grande dimension des exploitations. D'ailleurs, tant les entreprises valenciennes qui opèrent comme entreprises de services que les entreprises d'importation directe sont d'accord pour affirmer que les standards et les normes facilitent la communication entre les entreprises des deux hémisphères. Elles génèrent une proximité organisationnelle et culturelle malgré la distance physique. Les mécanismes de gouvernance et la nature du processus de création de réseaux mondiaux de production sont conditionnés par ce processus. En effet, pour de nombreux importateurs directs, la sécurité que leur apportent les certifications de respect des standards publics et privés est justement ce qui les a amenés à abandonner l'idée de réaliser des investissements productifs directs en Amérique Latine. 
C'est dans le domaine des variétés, du savoir-faire commercial et de certains traitements de post-récolte (déverdissage des oranges) que les entreprises espagnoles fournissent le plus d'informations aux producteurs d'Amérique Latine. A l'inverse, de plus grandes connaissances sont reconnues aux entreprises d'Amérique Latine (surtout celles d'Uruguay) et à celles d'Afrique du Sud en ce qui concerne les soins apportés aux fruits pendant la récolte et leur préservation jusqu'au lieu de destination. Les connaissances sont transférées lors des nombreuses visites annuelles réalisées par les deux parties, pendant les rencontres sur les foires et par des entretiens téléphoniques. De plus, il existe un courant continu de personnel universitaire et de chercheurs et professeurs qui font des courts séjours, des thèses doctorales et le master de culture d'agrumes de l'Université Polytechnique de Valencia (UPV) - Institut Valencien des Recherches Agricoles (IVIA). Ces visites sont aussi fréquentes de la part des chefs d'entreprise et des ingénieurs des centrales agrumicoles, visant surtout l'IVIA, les pépiniéristes valenciens et les entreprises les plus modernes. Les relations directes sont avantageuses pour les deux parties. Cela renforce la qualité des fruits et le positionnement de la centrale agrumicole valencienne. L'interaction entre les entreprises valenciennes et argentines permet d'accroître les connaissances sur le marché, la sécurité et le positionnement des entreprises argentines sur leurs propres marchés. Autrement dit, l'apprentissage qu'elles réalisent lors de cette collaboration directe avec les entreprises valenciennes, fortes d'une longue tradition exportatrice, leur permet d'améliorer leur relation avec d'autres clients, tels que des chaînes de supermarchés, différents types de distributeurs et intermédiaires ou d'autres centrales agrumicoles. Quant aux entreprises valenciennes, elles consolident leur positionnement sur le marché et entrent dans une logique d'organisation du secteur globale et intégrée de la production et de la commercialisation.

Cependant, il est nécessaire de noter l'asymétrie qui existe entre la fréquence et l'assiduité avec laquelle les chercheurs d'Argentine et d'Amérique du Sud font des séjours à l'IVIA, notamment pour leur programme d'obtention de variétés, et les voyages plus rares qui se font dans le sens contraire. On peut y voir deux raisons. Tout d'abord, la spécificité des recherches conduites par l'Institut National Technologique de l'Agriculture et l'Elevage argentin et l'absence d'un programme continu d'obtention de variétés dans ce pays et ses voisins peuvent expliquer le peu d'intérêt des chercheurs valenciens à se rendre dans ces pays. Ensuite, le lien étroit de l'IVIA avec le gouvernement valencien, via son Conseil de l'Agriculture du Gouvernement Valencien, peut amener cet organisme à refuser de coopérer avec des entreprises argentines ou sud-américaines dans la mesure où ces demandes sont parfois considérées comme pouvant être contraires aux intérêts de l'agriculteur valencien. La complémentarité des saisons entre les hémisphères Nord et Sud donne de la continuité à la production de fruits frais de différentes variétés d'agrumes. En raison de l'amélioration des transports, cette complémentarité a simultanément l'effet d'intensifier le commerce international, d'un côté, et, de l'autre, d'augmenter les pressions et les chances réelles de développement de stratégies globales, en vue d'une continuité annuelle dans l'approvisionnement du produit. Mais puisque cette complémentarité n'est pas parfaite, des superpositions entre variétés similaires ont lieu pendant quelques périodes et ont pour conséquence d'intensifier la concurrence entre l'Espagne et l'Amérique Latine. Cela permet de comprendre pour quelles raisons les centrales agrumicoles coopératives ne font pas pour la plupart d'entre elles d'importations directes en 
contre-saison. Cela explique également qu'un ensemble de normes qui réglementent la production et le commerce des agrumes puissent être à la fois un stimulus et une barrière pour le commerce international. Étant donné que le suivi et le renforcement systématique des contrôles phytosanitaires des importations à contre-saison constituent une autre priorité politique du CAPA (Gouvernement Valencien), le fait que l'IVIA par exemple participe à l'élaboration de ces normes conditionne les relations entre les systèmes de production et d'innovation agrumicole valencien et ceux d'Amérique Latine et, notamment, celui de l'Argentine. En somme, les particularités des problèmes régionaux et l'orientation des politiques publiques peuvent provoquer des segmentations, ou tout au moins limiter la fluidité de communication entre les systèmes agro-alimentaires.

\subsection{Les problèmes d'approvisionnement en continu d'un produit de qualité}

Depuis les cinq dernières années, la dynamique des importations d'oranges en contresaison est marquée par une baisse des produits venant d'Argentine au profit de l'Uruguay et de l'Afrique du Sud, du Chili, du Pérou ou encore de l'Australie. Ce recul est essentiellement dû à trois facteurs qui se renforcent entre eux.

En premier lieu, cette baisse est la conséquence des problèmes phytosanitaires rencontrés par les oranges argentines (particulièrement de la région du Nord-Est) et la rigueur avec laquelle les ports et les autorités valenciennes appliquent les contrôles sur ce fruit. En effet, certaines règles sur les fruits et les légumes en matière de sécurité alimentaire, de conditionnement, d'étiquetage et d'inspections phytosanitaires peuvent être spécifiées par chaque pays (Garcia Martinez et Poole, 2004). Dans un pays décentralisé comme l'Espagne, les gouvernements régionaux peuvent accroître encore cette spécificité. La plupart des entreprises interrogées soutiennent que les contrôles phytosanitaires constituent le principal problème généré par les importations en contre-saison. Bien que l'Uruguay (et même l'Afrique du Sud) n'y échappent pas, ce sont les expéditions provenant d'Argentine et du Brésil qui présentent les plus grosses difficultés.

En deuxième lieu, et de manière liée, le manque de coordination entre les secteurs public et privé argentins et le non-respect des normes par certaines entreprises (Rodríguez de Tappatá, 2003) ont conduit les opérateurs valenciens à craindre un manque de fiabilité, de sérieux et de rigueur de la part des entreprises argentines quant au respect des conditions établies.

En troisième lieu, enfin, les crises économiques et financières argentines auraient aggravé les deux problèmes mentionnés et elles auraient provoqué, du point de vue des centrales valenciennes, une tendance à la réduction des investissements destinés aux traitements et à la culture en général, entraînant en conséquence une dégradation de la qualité externe de l'orange argentine.

Tous ces problèmes provoquent une grande incertitude et posent des difficultés pour la mise en place d'un programme d'importations avec les producteurs et les centrales argentines. Pour cette raison, depuis quelques années, la plupart des centrales agrumicoles qui souhaitent réduire le « risque phytosanitaire » et entretenir une relation stable avec leurs fournisseurs préfèrent «travailler » avec les entreprises uruguayennes et/ou sud-africaines. A cet égard, il faut prendre en considération que l'Uruguay et l'Afrique du Sud ont réagi de manière totalement différente de l'Argentine. En Uruguay, la lutte rigoureuse contre le chancre et la concentration de 
l'offre dans deux grandes sociétés ou organisations (Urudor et Caputto) favorisent la sécurité et la stabilité des relations. En Afrique du Sud, le pays est passé d'une commercialisation initialement tout à fait contrôlée par l'Etat (Mercier et Tanguy, 2005), à une commercialisation réalisée par deux grandes sociétés privées, qui ont établi des relations directes et personnelles avec les centrales valenciennes.

Les problèmes associés aux contrôles phytosanitaires, mais aussi de qualité et d'incertitude, ont conduit les opérateurs argentins à explorer d'autres marchés, en Europe de l'Est et au Moyen Orient (Gonzalez Ojeda, 2003). En définitive, cette situation pourrait actuellement obliger de nombreuses entreprises argentines à rechercher des marchés moins exigeants et à « revenir » sur des circuits de vente beaucoup moins sûrs et rentables. A terme, une telle stratégie risque toutefois de les empêcher d'établir des rapports fructueux en termes d'informations, d'apprentissage et d'insertion dans des réseaux. En fait, il s'agit selon nous d'une régression de la «bonne » position obtenue pendant la deuxième moitié des années 1990 par les entreprises argentines.

Il existe cependant un petit nombre de grandes entreprises argentines exportatrices d'agrumes (essentiellement deux : Ledesma et San Miguel) qui continuent d'entretenir des relations stables avec les sociétés espagnoles et qui détiennent une part croissante des exportations argentines et des importations espagnoles. Ces entreprises approvisionnent aussi bien les centrales valenciennes qui opèrent comme sous-traitantes des centrales d'achat des grandes chaînes, que les centrales importatrices directes. Le fonctionnement de ces entreprises met en évidence l'importance discriminante, en ce qui concerne la compétitivité des exportations argentines sur les marchés européens plus exigeants, des facteurs indiqués ci-dessus : la qualité du produit et le sérieux du fournisseur. L'interaction sans doute croissante de ces entreprises (situées au Nord-Ouest de l'Argentine) avec la région productrice du Nord-Est, afin de pouvoir approvisionner les marchés avec les oranges plus tardives, de plus forte coloration et qui de ce fait ressemblent plus aux oranges valenciennes, pourrait stimuler sur ce territoire un développement plus rapide de nouvelles routines et formes d'organisation.

\section{Conclusion}

Cet article met en évidence que la croissance des exigences en termes de qualité, d'environnement et de sécurité alimentaire de la part des consommateurs et des chaînes de supermarchés ont provoqué un processus de réorganisation du système agrumicole valencien, tant du point de vue interne qu'en ce qui concerne ses relations externes. Cette réorganisation implique un processus de construction historique et sociale dont le résultat et les contours ne peuvent pas être établis a priori. La diffusion internationale de normes et de standards favorise la communication interne et externe entre les acteurs des systèmes agro-alimentaires. La diffusion de ces normes, cependant n'estompe pas les particularités régionales et les différences qui existent entre les différents systèmes concernés (Mercier et Tanguy, 2005). Dans notre cas, nous avons vu que certaines particularités politiques et économiques des systèmes agro-alimentaires peuvent limiter l'interaction et, par conséquent, la génération de synergies.

Dans le cas spécifique du système agro-alimentaire des agrumes de la Comunidad Valenciana, le caractère stratégique des centrales agrumicoles, le rôle central des innova- 
tions variétales, la politique du gouvernement régional et le type d'orientation des centres scientifiques et technologiques expliquent la forme précise adoptée par les réseaux de relations entre les différentes entreprises, et entre les entreprises et les institutions du système agro-alimentaire valencien. Les importations à contre-saison provenant d'Argentine renforcent la position réciproque des centrales agrumicoles des deux pays. Aux craintes que ce phénomène provoque dans le secteur de la production valencien, s'ajoutent les problèmes de contrôle argentin et les principales difficultés quant aux importations. Celles-ci ne proviennent pas des normes et des standards privés (Garcia Martinez et Poole, 2004) mais des contrôles phytosanitaires et de la qualité du produit. Dans ce contexte, les particularités régionales peuvent court-circuiter la communication au lieu de générer des synergies, malgré le flux important de personnes circulant d'Argentine vers la Comunidad Valenciana.

\section{Références}

Callon, M., 1991, Réseaux technico-économiques et irréversibilités, dans Boyer, R., Chavance B. et Godard, O. (dir), Les figures de l'irréversibilité en économie. Editions de l'Ecole des Hautes Etudes en Sciences Sociales, Paris: 195-230.

Cooke, P. Uranga, M.C. et Etxebarria, G., 1998. Regional systems of innovation: an evolutionary perspective, Environment and Planning A 30, 1563-1584/

Gallego, J.R., 2004. Noeuds technologiques localisés, apprentissage interrégional et dynamique des systèmes d'innovation. Le cas de la culture espagnole des agrumes, Les quatrièmes journées de la proximité, Marseille, 17-18 juin.

Garcia Martinez, M. et Poole, N., 2004. The development of private fresh produce safety standards: implications for developing Mediterranean countries, Food Policy 29, 229-255/

Gonzalez Ojeda, J., 2003. Análisis de la tendencia del mercado internacional de frutas cítricas. Informe Mensual de la Fundación Export. Ar, nº12.

Held, D., 2005. Un pacto global. La alternativa social-demócrata al Consenso de Washington. Taurus, Madrid.

Kaufmann, A. et Tödtling, F., 2001. Science-industry interaction in the process of innovation: the importance of boundary-crossing between systems, Research Policy 30, 791-804/

Mercier, D. et Tanguy, C. 2005. Entre homogéneisation par les normes et logiques d'action différenciées: la production d'oranges en Argentine et Uruguay, Économies et Sociétés 24,751-774/

Metcalfe, J.S., 1995. Technology systems and technology policy in a evolutionary framework, Cambridge Journal of Economics 19, 25-46/

Porter, M., 1991. La ventaja competitiva de las naciones. Plaza \& Janes, Barcelona.

Rallet, A. et Torre, A., 2005. Proximity and Localization, Regional Studies 39, 45-59/

Rodríguez de Tappatá, A., 2003. Estudios Sectoriales. Fruticultura de Exportación. Pomáceas y Cítricos Dulces. Oficina de la CEPAL-ONU en Buenos Aires.

Tomás Carpi, J. A. (dir), Banyuls, J., Cano, E., Contreras, J. L., Gallego, J.R., Picher, J.V. Such, J., et Torrejón, M., 1999. Dinámica industrial e innovación en la Comunidad Valenciana. IMPIVA, Valencia.

Torre, A., 2000. Economie de proximité et activités agricoles et agro-alimentaires. Eléments d'un programme de recherche, Revue d'Economie Régionale et Urbaine III, 407-426/ 
